\title{
Establishment of Paediatric and Neonatal Intensive Care Units at Patan Hospital, Kathmandu: Critical Determinants and Future Challenges
}

\author{
Adhikari $\mathrm{N}^{1}$, Avila $\mathrm{ML}^{2}$, Kache $\mathrm{S}^{3}$, Grover $\mathrm{T}^{4}$, Ansari ${ }^{5}$, Basnet $\mathrm{S}^{6}$ \\ ${ }^{1}$ Dr. Neelam Adhikari, MD (Paediatrics). Rector and Professor in Pediatrics, Patan Academy of Health Sciences, \\ Kathmandu, Nepal, 'Late Michele Lynn Avila, RN, MSN, CNS, CCRN. Lucille Packard Children's Hospital at Stanford \\ Hospital and Clinics, Palo Alto, CA, ${ }^{3}$ Saraswati Kache, MD. Assistant Professor of Paediatrics, Stanford University \\ School of Medicine, Palo Alto, CA, ${ }^{4}$ Theresa Grover, MD, Associate Professor of Paediatrics, University of Colorado \\ Denver School of Medicine, Denver, Colorado, 5 Imran Ansari, MD (Paed). Associate Professor Paediatrics, Patan \\ Academy of Health Sciences, Kathmandu, Nepal. ${ }^{6}$ Sangita Basnet, MD, FAAP Assistant Professor of Paediatrics, \\ Southern Illinois University School of Medicine, Springfield, Illinois
}

Address for Correspondence: Dr. Neelam Adhikari, E-mail: neelamadhikari@ gmail.com

\begin{abstract}
Introduction: Although preventive medicine and primary care are priorities in developing countries, they must be supported by appropriate care of sick and extremely sick children in the medical facilities. Lack of resources and absence of skilled physicians and nurses may lead to poor outcomes in critically ill patients. Intensive care of newborns and children is thought to be very expensive with a low cost-benefit ratio. This presentation discusses the critical factors that facilitated the establishment of PICU and NICU in an urban public hospital in Nepal, where a good standard of Level 2 care was already provided. Aims and objectives: A cooperative model of creation and transfer of technology from the West to a resource-poor country was envisaged. PICU and NICU with six beds each were established. Design and setting: The Nick Simons Foundation, USA provided financial support for design, building and furnishing of a new Mother and Child Wing at Patan Hospital. A generous grant of $\$ 300,000$ again by the Nick Simons Foundation helped equip the units. Donated equipments also included procedures, medication and storage carts. Methodology: A total of 22 volunteers, 21 from USA and one from Netherlands, were recruited to complete the three months of training. An extensive curriculum was prepared. The trainer team had monthly teleconferences and regular communications with the Chief of Paediatric Services and Nursing Director of Patan Hospital via e-mails and telephone. Responsibilities of volunteers and the host hospital were identified. Results: After 3 years of preparation, the project started in June 2009. All day lectures on topics in critical care, mock case scenarios, practical equipment training and simulated procedures led to the graduation of 60 nurses. Twenty five physicians were trained for three months. The expert team worked with the locals in preparing the units, arranging furniture and equipment, stocking carts, making inventory and preparing protocols. A protocol handbook was developed on topics such as mechanical ventilation, sedation, admission/discharge criteria, procedures and management of different disease states. Various charts such as nurse observation charts, notes by residents, procedure hand offs at change of shifts were designed and printed. Infection control practices and methods of sterilizing non disposable articles were identified and protocols written. At the end of three months the units were functioning with trained local manpower and reasonable modern equipment. Conclusion: Developing nations may not have enough resources to establish much needed critical care facilities. Developed countries can help by funding basic infrastructure and providing expertise in order to transfer knowledge and technology. Involvement in planning from the beginning and training at the host site is a preferred model of transfer of technology.
\end{abstract}

Key words: Paediatric Intensive Care Unit (PICU), Neonatal Intensive Care Unit (NICU), Patan Hospital 


\section{Introduction}

$\mathrm{M}$ ost children who become ill, even those with life threatening disorders, recover to lead satisfying and healthy lives after treatment. This is truer in developing countries, where the vast majorities of these illnesses are of infectious origin and are potentially treatable. This is also one of the main reasons why many young doctors choose to become paediatricians. Studies from developing countries have shown that lack of resources and absence of skilled physicians and nurses may lead to poor outcomes in critically ill patients ${ }^{1,2}$.

Nepal is one of the world's poorest nations and has a rapid population growth rate. About a third of its people are estimated to be living below the poverty line ${ }^{3,4}$. Access to care differs considerably according to geographical setting and other factors. The prevalence of malnutrition among children is high $^{5}$. It has a very high mortality rate for children aged below five years, estimated at 61 deaths per 1000 live births. Approximately $40 \%$ of population (i.e. 26 million people) is below 14 years of age. Neonatal Mortality Rate is $34 / 1000$ live births and infant mortality $48 / 1000$ live births. Only $44 \%$ pregnant women receive any antenatal care, and more than $80 \%$ deliver at home ${ }^{6}$.

\section{Critical care in developing countries}

The science of Paediatric Intensive Care has progressed significantly during the last four decades. Rapid advances in technology and knowledge have made this progress possible. Intensive care units are regarded as making a substantial contribution to the health of children in developed countries ${ }^{2}$.

There is always a question whether Intensive Care of Paediatric and Neonatal patients is appropriate in resource poor countries, where many sick children do not even have access to basic medical facilities. Even those women who do deliver in health facilities are unable to receive Level II neonatal care for their babies in most health facilities in Nepal. Intensive care requires allocation of space, adequate financing, equipment, staff, protocols of care, and infection control measures. Neonatal mortality could be reduced theoretically by adding NICUs at a few key hospitals. The National Neonatology forum recommends 30 NICU beds per million populations. Each bed should require 50 square feet per cradle and proper climate control. The largest expense is in equipment purchase, maintenance, and repair. Trained manpower is needed ${ }^{7}$ therefore small units could not make any major impact on mortality statistics in a developing nation ${ }^{8}$.
Preventive medicine and primary care are priorities in developing countries, which must be supported by appropriate care of sick and extremely sick children in the medical facilities. This is important not only to meet the expectation of parents and families of these children, but also to build their confidence in the preventive and primary care that is provided at the grassroots. Lack of resources and absence of skilled physicians and nurses may lead to poor outcomes in critically ill patients. However in addition to developing good non-intensive medical facilities all over the country, Intensive Care Units should be instituted in a slow, phased manner, with the well-equipped teaching hospitals taking the lead ${ }^{9}$.

Intensive care of newborns and children is thought to be very expensive and to have a low cost -benefit ratio. However, when the satisfaction of the family and the protection of long productive years that intensive care brings about are taken into account, this is found to be untrue ${ }^{8}$.

\section{Critical care in Nepal and at Patan Hospital}

There are very limited critical facilities in Nepal. Those that exist are mainly situated in large city hospitals, mostly in private ones. Patan Hospital is one of the largest hospitals in Nepal. It uses modern equipment and facilities to provide treatment for almost 320,000 outpatients and 20,000 inpatients every year. Patan Hospital staffs conduct more than 10,000 operations annually. It is one of two large hospitals (along with some other smaller ones) in Kathmandu Valley that accepts pediatric referrals and has paediatric inpatient facilities. An ICU for adult patients was established in the year 1998, which has been upgraded to a modern unit with 16 beds this year.

\section{Factors that created the Environment for PICU, NICU}

\section{A. Credibility through work and experience}

1. The hospital had 25 years of history of providing good and reputed medical care. Gradually the hospital had become a training hospital for Post Graduate Training for residents in different specialties. In the department of Paediatrics, the postgraduate training for General Practice and for Paediatrics started in the year 1984 and 1997 respectively, in collaboration with the Institute of Medicine, Tribhuwan University Hospital and later also with the National Academy of Medical Sciences. The standard of their training will certainly improve with rotation in properly equipped and managed intensive care facilities. 
2. Early Neonatal mortality rate had approached 10/1,000 live born babies and Perinatal mortality was $25 / 1000$ newborns ${ }^{10}$. Further mortality can only decrease with institution of level III care.

3. Some salvageable children were also dying because of unavailability of PICU. A 52-month audit of the pediatric service revealed sepsis/septic shock as the most common cause of death (74 deaths out of $207 ; 36 \%)^{12}$. Same audit also found meningitis as the most fatal (case fatality rate $6.5 \%)$. A majority of deaths (56\%) occurred within 48 hours indicating the acute nature of the illnesses. These two clinical conditions, as also many other infections, are potentially treatable provided that the most critical first few hours (or days) could be tided over with the provision of intensive care ${ }^{11}$.

4. Due to lack of PICU, a few older seriously sick children were sometimes admitted and treated in the adult ICU. But due to lack of appropriate sized ventilators, other equipment and nurses inexperience in management of critically sick children, the management used to be unsatisfactory.

5. The hospital has a good surgical team in the hospital but many pediatric patients had to be referred to other hospitals just because we did not have ICU back up and support.

6. Many wealthier families had started demanding a higher level of care for needy newborns and children. Intensive care, though already available at a few facilities was inadequate/ lacked quality/ was extremely expensive /combination of these.

\section{B. Expansion of Maternal and Neonatal health Facilities}

The workload in all departments of the hospital had increased considerably, and thus needed expansion. A generous donation by Jim and Marilyn Simons in memory of their son Nick (Nick Simons Foundation) allowed for the creation of a structure dedicated to women and newborn health, attached to the main hospital at Patan.

\section{Nepali diaspora in USA, willing to help Nepal}

A pediatric intensivist of Nepali origin, working and teaching in University School of Medicine, Department of Paediatrics, Springfield, Illinois USA had a deep desire to help in establishing a PICU in Nepal

\section{Support from Friends of Patan Hospital}

There had been continuing help from Friends of Patan Hospital (FOPH) ${ }^{12}$, a nonprofit, tax-exempt organization, assisting Patan Hospital with fund raising, provision of equipment and training of personnel, since 2002. Ms. Michele Avila, one of the two Program Coordinators; a nurse practitioner working at Stanford School of medicine, Department of Pediatrics Illinois was the then Vice President of FOPH.

\section{E. American academician's desire of helping a resource poor country.}

Dr. Sangita Basnet got together a team of trainers by posting a message on the SCCM website. This twenty two member expert team consisted of 6 physicians, one paediatric nurse practitioner, 11 nurses, 3 pharmacists and one respiratory therapist- all whom were specialists in critical care. Late MS. Michele Avila, Dr. Sangita Basnet and Dr. Theresa Grover provided leadership. All but three of these volunteers spent at least two weeks on the project; over half spent three or more weeks. One volunteer, Titia Boers, a nursing educator, spent twelve weeks in Nepal and contributed extensively towards setting and stocking of units in addition to education.

The volunteers donated their time books, other teaching material and also were responsible for arranging their own airfare. The staffing was so designed that everyone's term overlapped for at least a week and everyone was already familiar with the part they were to play in the training.

\section{F. Support of Patan Hospital administration.}

The hospital pledged to provide modest lodging, food and housekeeping at the hospital campus to the volunteers during their stay. Permission to work in Nepal was obtained from the Nepal Medical Council and Nepal Nursing Council through Patan Hospital. Other necessary arrangements of housekeeping, site seeing etc. were facilitated by the hospital secretary.

\section{Material and Methods (Process)}

A cooperative model of creation (funding, planning and execution of training) and transfer of technology from West to a resource poor country was envisaged.

The pediatric consultants recognized the need for a Neonatal Intensive Care Unit (NICU) in the new Mother and Child wing and a space was dedicated for this. At the same time as there was no facility in the hospital for intensive care of seriously sick children, it was felt appropriate to establish a Paediatric Intensive Care Unit (PICU) too. The basic premise was that both facilities would require many common principles in planning, equipping and training of medical staff.

PICU and NICU, with six beds each were planned. 
The design was planned in close collaboration with $\mathrm{OZ}$ architects, USA and local medical and administrative staff. A building committee of stakeholders was established, which met every week. Recommendations for organization of Level III neonatal services in developing countries ${ }^{8,13}$ special care units were followed in planning equipment and manpower for NICU and to some extent for PICU too.

Local vendors were contacted for necessary equipment. Experience of some Nepali paediatricians, an Indian neonatologist and an intensivist in USA helped in choosing, ordering and purchase of the equipment. While selecting equipment, due consideration was given to cost benefit ratio, ease of use, maintenance, biomedical support of the company and adaptability to the hospital's infrastructure. Although most equipment was new, some used donated equipment, procured and shipped by FOPH, USA was also used.

Besides communicating through innumerable emails and telephonic communications, Dr. Sangita Basnet and Ms. Michele Avila (Vice President of FOPH) made two visits each to Kathmandu to discuss and collaborate with local staff and administration. The strength of the present medical staff and available facilities at Patan Hospital were assessed. Periodic teleconferences between the trainers regarding curriculum and other arrangements were conducted. The need to move from shared nursing responsibility, prevalent at that time to that of instituting primary nursing in the ICU was identified to provide optimal patient care.

All activities required considerable time for preparation since all involved also had full time jobs.

\section{The Curriculum}

A rigorous three-month curriculum was developed, which consisted of the following components:

1. Provision of Advanced pediatric life Support (PALS) and Neonatal Resuscitation Program (NRP) and Safe Care- Sugar- Temperature-Airway-Blood Pressure-Labs-Emotional Support (STABLE) courses.

2. Practice of mock scenarios with dummies and other equipment.

3. Didactic sessions with an organized daily lecture series: physician and nurses, with many lectures being combined.

4. Bedside clinical support to physicians and nurses during day and night.

5. Daily case conferences.
6. Morbidity and mortality discussions.

7. Trouble-shooting: to discuss unanticipated complications.

8. Computer based learning: this included the delivery of all didactic lectures on a Beta Microsoft workspace to which all participants had passwords.

9. Paediatric ICU handbook with 28 chapters of relevant ICU topics regarding patient management was written by some of trainers as protocol book.

10. Order sets for commonly encountered disease processes.

11. Policies and procedures for basic ICU patient care guidelines, e.g. extubation criteria

12. Admission criteria to both PICU and NICU appropriate for setting of Patan Hospital were discussed and finalized.

This resource material was downloaded in a computer in the units with a printer facility. The two pharmacists designed programs for drug (commonly used drug in ICU setting) dosages and method of constitution for each weight. These sheets for a particular weight of the patient could be printed and attached to the patient's chart. In absence of a designated pharmacist for ICUs, (as is common practice in developing countries), this has been greatly useful for ease of use and minimization of medical errors in drug delivery.

The main focus of training was on strengthening and reinforcing assessment skills, 24 hour monitoring, and critical thinking. Important concepts such as pain control and sedation, respiratory management including noninvasive and invasive mechanical ventilation, blood gas analysis, review of physiology and support of different organ systems, and early sepsis management were introduced and elaborated. Emphasis was placed on teamwork, infection control practices and organization. Both nurses and physicians learned about assembly, use, care, and storage of equipment, medication calculations and errors, procedures and general nursing care in the units. Fortunately the hospital staff was already strong in some of these spheres. The hospital maintenance team and the vendors who supplied the equipment provided excellent help.

Nepali participants consisted of pediatric consultants, registrars, nurses and pharmacists. The expertise brought by the host country team members consisted of their extensive knowledge of the local disease processes, limitations of the local resources, and the capacity to adequately manage patients within limited constraints. 


\section{Implementation}

A foundation course of full day training was held for three weeks before opening the units. PALS, NRP and STABLE trainings were provided. Admission criteria, easy to use protocols for indication and use of ventilators, extubation, pain and sedation (ICU and procedural), and sedation weaning were prepared. Mock drills practiced various procedures, and emergency management of various disease conditions including hypoxemia, asthma, sepsis, shock etc. Locally suitable charts were prepared for nursing recording, resident sign out, ventilator management, and pharmacy. Medication, code and procedure carts were prepared and stocked. Inventories were prepared for restocking and smooth functioning of the units.

After three years of preparation, the fully functional units opened in July 2009 for patient care. On the first day, a 35-week gestation newborn born to a mother with severe eclampsia was admitted to the NICU. He had severe respiratory distress with refractory hypoxemia (saturation 65\%) on bubble continuous positive airway pressure (CPAP). Emergent endotracheal intubation was done and baby placed on ventilator. The baby was transferred to Level II care after seven days and later sent home. On the same day a 2-year-old boy with severe $40 \%$ burns was transferred from surgical ward to the PICU with pseudomonas sepsis and shock (hypovolemic and septic). He also survived with PICU care for 21 days and was later cared for in Pediatric ward for a week before discharge to home.

\section{Results}

At the end of three months, fully functional PICU and NICU with six beds each were created. The units have facilities for noninvasive and invasive monitoring, ability to ventilate five children and four newborn patients at one time. The local medical and nursing staff feels comfortable in managing common problems in $\mathrm{PICU}$ and NICU. Regular records of admitted patients are kept and monthly audits are performed. There are dedicated nurses for the units, who sometimes also work in Level II nurseries depending upon the work load. Primary nursing care model is followed. Nurse to patient ratio is kept at 1:2.

A reasonable number of library books, a hand book for PICU, protocols, observation charts, electronic versions of teaching learning materials used in the training saved in a house computer for immediate consultation, exist within the units. As previously described programs for drug dosages (for commonly used drugs in ICU setting) and method of constitution for each weight, are very helpful in work and minimizing medical errors. Indications for admission to PICU and NICU are finalized and followed. Both the units now have internet facilities. Access to Up To Date ${ }^{14}$ is available within both the units.

Most patients pay for their own care fully or at least partially. Needy patients get help from the hospital social services. Attempts are being made to raise more finances for help for the needy, but this has not been easy and adequate. A PICU/NICU fund is established for poor patients.

Time is required for reflecting on the process and considering strategies for improvement. We will be analyzing our experience with delivery of service, soon.

\section{Discussion}

Paediatric and neonatal intensive care is a relatively new medical specialty that has shown marked growth around the world over the last three decades. It is even newer in developing countries. Compared to the developed countries, children admitted to the PICUs in developing countries have higher mortality. Medical paternalism is more often desired and has a strong influence in the decision-making process offered to terminally ill patients in developing countries ${ }^{8}$. Multiorgan Dysfunction Syndrome (MODS) in children usually occurs early, and sepsis, which is the leading cause of MODS in developing countries, increases mortality ${ }^{16}$. Further sick children in resource-constrained countries present late to the hospital. Studies from developing countries have shown that lack of resources leads to poor outcomes in critically ill patients. More importantly, the skill and availability of physicians and nurses may affect patient outcome even more than technology $y^{15,16,17,18}$. Keeping this in mind, continued in service training to nurses and doctors and regular visits from some of the members of the training team is planned.

Triage of critically ill children and neonates is essential to ensure that intensive care is only offered to those who are most likely to benefit. Providing intensive care to patients who have dismal prognosis or only to prolong the life of patients, who have vegetative existence, is inappropriate in developing countries. Scoring systems that predict the risk of mortality for children in an intensive care unit (ICU) are needed for the evaluation of the effectiveness of paediatric intensive care. The Paediatric Risk of Mortality (PRIM) ${ }^{19}$ and the Paediatric Index of Mortality (PIM) scores ${ }^{20}$ have been developed to predict mortality among children in the ICU. Some recent studies have also evaluated these in developing countries too. Preliminary results of an ongoing evaluation of morbidity stratification of children 
admitted to the pediatric ward at Patan Hospital before the Pediatric ICU was established shows that over $15 \%$ of patients are admitted with a severity of illness that would require intensive care unit management according to the Signs of Inflammation in Children that can Kill $(\mathrm{SICK})^{21}$ score and Paediatric Risk of Hospital Admission Score (PRISA) ${ }^{22}$. This study is yet to be published.

The estimation of cost-effectiveness of pediatric critical care services is an extremely relevant issue for both developing and industrialized nations. Paediatric critical care is expensive and the long-term outcomes are still relatively unclear. From the perspective of patients who receive the benefits of these services, there may be little controversy regarding cost-effectiveness. However, the issue becomes very complex when attempts are made to identify which patients will benefit most. This also needs to be considered while developing public policy when decisions for allocation of limited resources need to be made within health care systems, and choices need to be made between the provision of health care and other public services ${ }^{2}$. Proponents of Paediatric and Neonatal Intensive Care argue that this is highly cost effective as saving the life of a young child leads to survival and productivity of over five decades as opposed to intensive care of a seriously sick adult with cancer or heart disease, which leads to a much shorter productive life $^{2}$.

Several organizational aspects of PICU /NICU services are very important, effecting quality of care and outcome. Concentration of resources in tertiary centers, regionalization of care and employment of full time intensivist/s among others, can help quality of care and outcome $23,24,25$.

For training of Intensive Care Units, some other models are also in place and are very useful in under resourced countries. Under the direction of Dr. Jeffrey Burns, Chief of the Division of Critical Care Medicine at Children's Hospital Boston, a video library has been established. The videos on this website are part of a pilot project to bring paediatric intensive care resources to anyone, in anyplace across the world by creating a web-based video library of notable clinicians speaking on "the full range of topics essential to paediatric critical care medicine ${ }^{26 "}$.

\section{Challenges and Further Implications}

\section{Ethical dilemmas}

We are facing many ethical dilemmas as in other ICUs. The main ones are inability for the patients to clear the bills in time, families being at loss to make important decisions on continuing or discontinuing treatment and wanting treating physicians to make those for them etc.

\section{Continued in service training}

Since the inception of the units, a repeat on the job training has been given to the doctors by local consultants and similar training will be given to the nurses soon. Our main challenges are continuing to develop and maintain an inter-professional workforce that is appropriately skilled and motivated. We want to ensure that the staff has access to use of necessary material for their own educational and training needs, and want to maintain yearly mandatory training to all staff. We plan to establish Work Based Learning (WBL) and coordinate joint nursing and medical education and development.

We realize that any training provided is best when it is hands on, rather than based only on observation. Our relationship with the founding group, their wish to visit and support at least once a year and telemedicine links with SAARC telemedicine program and others in the future will be extremely helpful for continuing education and learning. For the intensive care units, establishing a healthy work environment is not only about the sterility or low rates of infection, but rather forming respectful, professional relationships among its team members. This is especially true when two teams are from different cultures.

\section{Unsafe Transport}

At present we are getting referrals not only from Kathmandu but also from all over Nepal. These patients are not accompanied by any health worker and do not receive any medical treatment on the way. Transport incubators for small babies are not available; we have had two patients brought to our hospital intubated and hand ventilated by bag and mask by the family in a car from a distance of $200 \mathrm{kms}$ ! Outcome of patients transported in such hazardous way will lead to poor outcome.

\section{Staffing}

Four new Paediatricians have been employed and employment of one more is planned in near future. Two promising residents have been sponsored and are undergoing training to be paediatricians. Some of these young paediatricians will be sponsored to specialize in Paediatric Intensive Care. At present, the workload of the department, which also manages a busy General Paediatric Service and busy Level I and Level II neonatal units, is heavy. It is expected to get easier with the planned recruitment of new personnel. 
Since the inception of the units, a repeat on the job trainings are periodically given to the doctors and nurses by local consultants.

Unfortunately four of our trained nurses have already left to live and work in Western countries. Training and retention of trained staff is going to be a big challenge. In 2005, the American Association of Critical Care Nurses (AACN) introduced six tenets that are necessary to establish and sustain healthy work environment (HWE) in a health care setting: skilled communication, true collaboration, effective decision making, appropriate staffing, meaningful recognition and authentic leadership ${ }^{27}$. Our nurses will have to strive very hard to achieve these.

\section{Financial concerns}

Costs of maintenance of equipment and care of poor patients are financial concerns.

\section{Conclusion}

We have presented a cooperative model of creation (funding, planning and execution of training) and transfer of technology from the West to a resource poor country, which was successfully implemented in Nepal. There are critically ill children in low income countries, in fact, in greater numbers than developed nations. Preventive and good curative intervention should be the priority; however, emergency and critical care facilities should also be developed proportionately. It should be every child's right to have access to appropriate health care including reasonable critical care management. Wealthy nations can help in making this a reality by funding the infrastructure and providing expertise and technological help. However, good collaboration and respect for the people and culture of the developing nation is essential for success.

We are at present helping in training of Paediatricians who will work in different hospitals of the country. It is hoped that continuing education and training at this teaching hospital will have a ripple effect and disseminate the concepts of intensive care and a higher vigilance for critical illness to other hospitals and, eventually, rural Nepal. We also hope to be able to help others in creating similar facilities.

\section{Acknowledgement/Funding}

We are grateful to Nick Simons Foundation for their generous donation. We thank America Nepal Medical Foundation (anmf.net) for their support and funding for travel of some of the volunteers and donation to PICU/
NICU poor patients'fund. We thank Beena Kamath, Jason Katz, Gary Lee, Jennifer Norgaard, Leslie Williams, Beth Case, Kwai Mak, Melanie Chan, Vanessa Darrell, Jeanette Cox, Sandra Miller, Vicky Albit, Marilyn Castaneras, Jacqueline Gianelli, Tracy Pablo, and Titia Boers, for their dedication and time to make this project successful. We gratefully acknowledge help of Mr. Fred Langworthy, Madhav Dhungana from maintenance department of Patan Hospital. Last but not the least our heartfelt thanks to Dr. Rajesh Gongal, the then director of Patan Hospital and nursing director, Mrs. Ram Shova Risal.

Conflict of Interest: None

Permission of IRB: Yes

\section{References}

1. Bhal S, Tyagi V, Kumar N, Sreenivas V, Puliyel JM. Signs of Inflammation in Children that can Kill (SICK score): Preliminary prospective validation of a new noninvasive measure of severity-of-illness. $J$ Postgrad Med 2006; 52:102-105.

2. Dunser M, Baelani I, Ganbold L. A review and analysis of intensive care medicine in the least developed countries. Crit Care Med 2006; 34: 1234-1242.

3. World Bank. Nepal at a glance. Available at: http://devdata.worldbank.org/AAG/npl_aag.pdf. Accessed 23 September 2008.

4. United Nations Development Programme (UNDP). Millenium development goals: needs assessment for Nepal. Kathmandu: Government of Nepal/ UNDP, 2006.

5. Ministry of Health and Population. Nepal demographic and health survey 2006. Government of Nepal, 2006.

6. Central Bureau of Statistics. Nepal in figures 2007. Kathmandu: Government of Nepal, 2007.

7. Norms for accredition of level III Special Care Neonatal Units. Neonatology Forum of India (NNF) Available at: http://www.nnfi.org/normslevel3.html Accessed: 10th November 2010.

8. Ramesh C. Sachdeva. Intensive care- a cost effective option for developing countries? Indian $J$ Pediatr 2001;68(4):339-344.

9. Fernandez A, Mondkar JA. Status of neonatal intensive care units in India. J Postgrad Med. 1993; 39 (2):57-5. 
10. Ansari I, Adhikari N. Perinatal Mortality at Patan Hospital. J Nepal Health Research Council 2003; 3: $42-45$.

11. Ansari I. Trends of Childhood Diseases in Patan Hospital. Presented in the XIV Conference and published in the Souvenir of Nepal Pediatric Society 2008.

12. Friends of Patan Hospital. Available at: http://www. foph.org/index.htm. Accessed 14 November 2010.

13. Singh $M$ and Paul VK. Organization of neonatal services in developing countries. Indian $J$ Pediatr 1995:62:139-144.

14. Upto Date. UpToDate is an evidence-based, peer-reviewed information resource available via the Web and mobile devices. Available at: http:// www.uptodate.com/home/index.html. Accessed 12 November 2010.

15. Tantaleán, José A. MD; León, Rosa J. MD; Santos, Alejandro A. MD; Sánchez, Eduardo MD. Multiple organ dysfunction syndrome in children.Pediatr Critical Care Med. 2003;4(2):181-185.

16. Jefferson PP, Eduardo S, Pedro CG, Ricardo CB. The burden of pediatric intensive care. Pediatr Resp Reviews 2005;6(3):160-165.

17. Earle M Jr, Martinez Natera O, Zaslavsky A, Quinones E, Carrillo H, Garcia Gonzalez E, Torres A, Marquez MP, Garcia-Montes J, Zavala I, GarciaDavila R, Todres ID. Outcome of pediatric intensive care at six centers in Mexico and Ecuador. Crit Care Med 1997;25:1462-1467.

18. Pearson G, Shann F, Barry P, et.al. Should pediatric intensive care be centralized? Lancet 1997; 349:1213-1217.

19. Naanings B, Ab-Hanna A, de Jorge E. Applying PRIM (Patient Rule Induction Method) and logistic regression for selecting high-risk subgroups in very elderly ICU patients. Int $J$ Med Inform 2008;77(4):272-9.

20. Pediatricex of Mortality (PIM): A mortality prediction model for children in intensive care. Intensive Care Med 1997;23(2);141-2.

21. Tygai V, Kumar N, Sreenivas V, Puliyel JM. (2006) Signs of Inflammation in Children that can Kill (SICK score): Preliminary prospective validation of a new non-invasive measure of severity of illness. J Postgrad Med 52:102-5.

22. Chamberlain JM, Patel KM, Pollack MM. The Pediatric Risk of Hospital Admission Score. (PRISA). A Second-Generation-Severity-of-Illness Score for pediatric emergency patients. Pediatrics 2005;115:388-395

23. Pollack MM, Alexander SR, Clarke $\mathrm{N}$ et al. Improved outcomes from tertiary center pediatric intensive care: a statewide comparison of tertiary and non tertiary facilities. Crit Care Med. 1991; 19:150-159.

24. Watson RS. Location, regionalization and outcome in pediatric critical care. Curr Opinion Crit Care 2002;8:344-348.

25. Pollack MM, Kaltz RW, Ruttiman UE, Getson jr. Improving the outcome and efficiency of intensive care: the impact of an intensivist. Crit Care Med 1988;16:11-17.

26. PICU without walls. Children's Hospital Boston. Available at: http://www.childrenshospital.org/ clinicalservices/Site2248/mainpageS2248P0.html. Accessed 13 November 2010.

27. American Association of Critical Care Nurses. AACN Standards for establishing and sustaining Healthy Work Environments, A Journey to Excelence 2005. Available at: http://www.aacn. org/WD/HWE/Docs/ExecSum.pdf. Accessed 13 November 2010.

\section{How to cite this article?}

Adhikari N, Avila ML,Kache S, Grover T, Ansari I, Basnet S. Establishment of Paediatric and Neonatal Intensive Care Units at Patan Hospital, Kathmandu: Critical Determinants and Future Challenges. J Nepal Paedtr Soc 2011;31(1):49-56. 